Recepción: 01 / 03/ 2018

Aceptación: 15 / 05 / 2018

Publicación: 07 / 07 / 2018

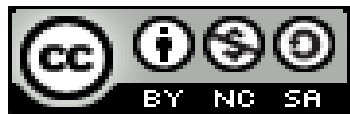

Ciencias Económicas

Artículo de Investigación

\title{
Producción y comercialización del pimiento e incidencia socioeconómica
}

Pepper production and marketing and socioeconomic impact

Produção e comercialização de pimenta e impacto socioeconômico

Carlos J. Cañarte-Bello I

ing_agrcjcb@hotmail.com

Tomas R. Fuentes-Figueroa II

tomas.fuentes@unesum.edu.ec

Bienvenido M. Vera-Tumbaco III

max-vera@hotmail.com

Narciso Fernando Augusto Ayón-Villao IV

fernando.ayon@unesum.edu.ec

Correspondencia: ing_agrcjcb@hotmail.com

I. Diplomado en Autoevaluación y Acreditación Universitaria, Ingeniero Agrónomo, Universidad Estatal del Sur de Manabí Jipijapa, Ecuador.

II. Magister Gerencia Educativa, Ingeniero Agropecuario, Universidad Estatal del Sur de Manabí Jipijapa, Ecuador.

III. Universidad Estatal del Sur de Manabí. Jipijapa, Manabí, Ecuador. Docente investigador de la Facultad de Ciencias Naturales y de la Agricultura. Carrera Agropecuaria, Universidad Estatal del Sur de Manabí Jipijapa, Ecuador.

IV. Magister en Agroecología y Agricultura Sostenible, Ingeniero Agrónomo, Docente Titular UNESUM, Coordinador Carrera Ingeniería Agropecuaria, Universidad Estatal del Sur de Manabí Jipijapa, Ecuador. 


\section{Resumen}

El estudio estableció el volumen de producción e identificó los sistemas de mercadeo que utilizan los productores de pimiento en la comercialización; y la incidencia económica que ha originado la explotación. La investigación se ejecutó en la Parroquia Puerto Cayo. Del Cantón Jipijapa. Provincia de Manabí, durante los meses de enero hasta julio de 2009.

El examen de la información obtenida basada en las encuestas realizadas a horticultores y comercializadores de pimiento en el área de investigación, se proceso estadísticamente a través de porcentaje; permitió determinar la baja producción en el segundo semestre del año. Al mismo tiempo que no mantienen una coherencia de las actividades administrativas en correspondencia con las actividades prácticas de campo. Del mismo modo se identificó que la mayoría de los agricultores utilizan el canal de comercialización, agricultor-mayorista-minorista-consumidor; produciendo como resultado que el horticultor reciba un precio bajo en relación al alto costo que paga el consumidor final.

Intrínsecamente en la investigación se analizó que el desarrollo económico de este sector productivo está sujeto a varios factores como: la experiencia, la capacitación, la disponibilidad de recursos, el área de siembra, el material vegetal, la disponibilidad de mano de obra capacitada, el costo de producción, así como el precio de venta del pimiento y otros factores naturales que pueden incidir de manera positiva o negativa en esta actividad productiva. Definitivamente el trabajo de campo se llevo a efecto en los recintos Puerto La Boca y Cantagallo.

Palabras claves: Producción, comercialización, pimiento, incidencia socioeconómica. 


\section{Abstract}

The study established the volume of production and identified the marketing systems used by pepper producers in commercialization; and the economic impact that exploitation has caused. The investigation was carried out in the Puerto Cayo Parish. Of the Canton Jipijapa. Province of Manabí, during the months of January to July 2009.

The examination of the information obtained based on the surveys made to horticulturists and marketers of pepper in the research area, is statistically processed through percentage; allowed to determine the low production in the second semester of the year. At the same time they do not maintain a coherence of administrative activities in correspondence with practical field activities. In the same way, it was identified that the majority of farmers use the marketing channel, farmerwholesaler-retailer-consumer; producing as a result that the horticulturist receives a low price in relation to the high cost paid by the final consumer.

Intrinsically in the research, it was analyzed that the economic development of this productive sector is subject to several factors such as: experience, training, availability of resources, planting area, plant material, availability of skilled labor, production cost, as well as the sale price of the pepper and other natural factors that can have a positive or negative impact on this productive activity. Definitely the field work was carried out in the Puerto La Boca and Cantagallo enclosures.

Keywords: Production, marketing, pepper, socioeconomic impact. 


\section{Introducción.}

En Ecuador habían 891 hectáreas de pimiento sembradas en monocultivos por 1,734 Unidades de Producción Agropecuarias (UPAs), según el Censo Agropecuario 2000; otras 79 ha se sembraron en cultivos asociados. La producción fue de 5,000 toneladas. Más de la mitad de la superficie se encuentra en las provincias de Manabí y Guayas, en los meses de verano (entre Julio y Enero). (Holguin Mora, P. y Romero, E. 2002.)

La inexperiencia del manejo del cultivo se debe a la inexistencia de mano de obra calificada a pesar de estos problemas los productores siguen sembrando el pimiento con costosos esfuerzos, la disminución en la aplicación de tecnologías se debe al escaso apoyo de extensión y orientación de parte de las instituciones del estado, esto hace que los cultivadores tengan problemas económicos en el desarrollo productivo al no poder tener acceso a créditos por las elevadas tasas de interés.

Los productores de pimiento también enfrentan problemas en el proceso de comercialización ya sea por la oferta como por la demanda del producto. Así como los diferentes canales por los que atraviesa la hortaliza para llegar al consumidor. Lo que ha dado lugar a que muchos productores en determinado momento hayan reducido la superficie de siembra o abandonan el proceso de producción, generándoles problemas económicos al no haber un proceso continuo a fin de mantener el mercado, así como el nivel de producción, causando desempleo, disminución de rentabilidad y el incremento de la pobreza con lo que se restringe el desarrollo de la zona.

Según Linares O. Heladio (2004), el éxito del pimiento, radica en que es un cultivo con tres destinos de consumo: pimiento en fresco, para pimentón y para conserva. La demanda de los mercados de pimientos frescos durante todo el año, ha crecido espectacularmente y ha tenido como consecuencia el desarrollo del cultivo. El manejo postcosecha, es muy importante para preservar las 
condiciones del fruto, ya que en ocasiones, se pierde precio por el mal manejo y las malas condiciones de almacenaje.

La investigación permitió establecer el volumen de producción e identificar los sistemas de comercialización que aplican los productores de pimiento; como también la incidencia económica que ha ocasionado la explotación en el desarrollo socio-económico tanto local, regional y nacional.

\section{Materiales y métodos.}

Como objeto de estudio para esta investigación se considero a la población de los recintos Puerto La Boca y Cantagallo de la parroquia Puerto Cayo, del Cantón Jipijapa, Provincia de Manabí, que es de (568), habitantes. El tamaño de la muestra fue de 235 personas en total, de las que se encuestaron al azar: 88 en Puerto La Boca, y 147 en Cantagallo. Para la realización de la presente investigación se dispuso de los recursos, tanto humanos como materiales y financieros que fueron factores importantes en desarrollo de la indagación. Además se fortaleció la investigación con información de Libros, revistas, folletos y boletines.

Para el desarrollo de la presente investigación se planteo un estudio tipo cuasiexperimental apoyado en la concepción filosófica hermenéutica, descriptivo basado para la recopilación de la información en actividades de observación, entrevistas, encuestas, investigación documental e investigación de mercado, sobre el entorno de compra-venta en relación a los canales de comercialización del pimiento desde el productor hasta el consumidor en el Cantón Jipijapa.

En el presente estudio se utilizo las técnicas de observación y encuesta. La observación se utilizo en toda la investigación, porque a través de ella se pudo puntualizar la información recopilada de fuentes primarias y la encuesta fue aplicada a los productores, comercializadores y a 
las personas involucradas en la investigación. Se utilizo además instrumentos de medición, como el cuestionario de preguntas para las encuestas dirigidas a las personas involucradas en esta investigación.

Para el desarrollo de la investigación se realizo una exploración de campo, que sirvió de base para ampliar las generalizaciones del tema, al mismo tiempo permitió conocer con mayor claridad y objetividad la importancia del estudio de la producción y comercialización de pimiento. Toda esta información fue recopilada en base a las encuesta mediante un formulario de preguntas aplicadas a los cultivadores o personas que se dedican a la producción de pimiento, así como la información bibliográfica que es una fuente muy indispensable en este trabajo investigativo.

En la investigación se realizo el proceso estadístico a través de porcentaje, con la finalidad de observar tendencias fundamentales que nos llevo a determinar las conclusiones efectivas del trabajo de investigación y así dar cumplimiento a los objetivos propuestos.

Tradicionalmente el Ecuador ha producido pimiento para el mercado local todo el año, con picos altos en los meses de Junio a Diciembre. www.dspace.espol.edu.ec

La producción del pimiento para el mercado interno se da la mayor parte en verano porque en esta temporada la incidencia de lluvias es menor, para evitar problemas de drenaje, así como plagas y enfermedades. El periodo de mayor producción de pimiento en la Costa es entre Julio y Enero, en donde la demanda de la Costa se satisface con la producción de esta misma zona; en los meses restantes la demanda es cubierta con la producción de la provincia de Loja y Manabí, que en el invierno proveen al mercado pimiento. Según la siguiente tabla elaborada por (Holguin Mora, P. y Romero, E. 2002): 
Carlos J. Cañarte-Bello; Tomas R. Fuentes-Figueroa; Bienvenido M. Vera-Tumbaco;

Narciso Fernando Augusto Ayón-Villao

\begin{tabular}{|c|c|c|c|c|c|c|c|c|c|c|c|c|}
\hline PROVINCIAS & Ene & Feb & Mar & Abr & May & Jun & Jul & Ago & Sep & Oct & Nov & Dic \\
\hline CAÑAR & & & & & & & & & & & & \\
\hline CARCHI & & & & & & & & & & & & \\
\hline EL ORO & & & & & & & & & & & & \\
\hline ESMERALDA & & & & & & & & & & & & \\
\hline GUAYAS & & & & & & & & & & & & \\
\hline IMBABURA & & & & & & & & & & & & \\
\hline LOJA & & & & & & & & & & & & \\
\hline MANABI & & & & & & & & & & & & \\
\hline PICHINCHA & & & & & & & & & & & & \\
\hline
\end{tabular}

Tabla elaborada por Holguin Mora, P. y Romero, E.

Se utilizo como referencia esta tabla que demuestra que en otras zonas del país, el clima permite cosechar pimiento entre los primeros meses del año, de modo que los productores puedan aprovechar los mejores precios.

Los cultivadores que se dedican a la siembra y cosecha de pimiento son agricultores que tienen una educación promedio basada en los primeros años de primaria, cultivan la mayor parte del área de pimiento, y carecen de tecnología, empleando sus conocimientos aprendidos de generación en generación. El rendimiento promedio en la producción de pimiento es de 13.8 t/ha. La productividad juega un papel importante en la producción de una hortaliza, ya que a un mayor nivel 
de productividad se logran menores costos y mayores serán los ingresos netos del agricultor.

(Holguin Mora, P. y Romero, E. 2002).

En la península de Santa Elena se cultiva el híbrido Quetzal, a la variedad hay notorias diferencias, el primero demanda por hectárea de $\$ 500.00$ a $\$ 600.00$ más de costo por el valor de la semilla, pero compensa por el tamaño del fruto tolerancia a fusarium, más duración poscosecha y una prolongación de su ciclo productivo "una ventaja en producción del $30 \%$ cubre a la mayor inversión", este pimiento verde de tres punta, de 15 a $17 \mathrm{~cm}$. de largo, de $5 \mathrm{~cm}$. de diámetro y una producción de 1.200 sacos de $35 \mathrm{~kg}$. Es lo que el mercado nacional demanda, www.eluniverso.com $(2002-2003)$.

El Salvador es otro híbrido que se siembra en la península de Santa Elena, su periodo vegetativo es prolongado de 150 a 180 días y la cosecha que comienza a los 90 puede rendir hasta 1.500 sacos de $30 \mathrm{~kg} / \mathrm{ha}$., la uniformidad del fruto es otra cualidad y sobre todo el grosor de sus paredes (3.5mm a 4mm) le da una larga vida poscosecha, www.eluniverso.com $(2002-2003)$.

Las casas comerciales determinan la producción de sus materiales vegetales con experiencias obtenidas en lugares de experimentación en el campo, es así, como la casa Comercial Agripac S.A. determina un rendimiento de $30.000 \mathrm{~kg} / \mathrm{ha}$. Para el pimiento hibrido "Quetzal" (17). Donoso y Asociados S.A. estima una producción de $40.000 \mathrm{~kg} / \mathrm{ha}$. para el hibrido Bengal (18). Su cosecha dura de 2 a 4 meses con flujos fuertes de cosecha cada mes. Se hacen de uno a dos cortes por semana. Los rendimientos puede alcanzar los $48000 \mathrm{~kg} /$ ha Se recomienda realizar la cosecha utilizando tijeras o cuchillos. www.dspace.espol.edu.ec 
Carlos J. Cañarte-Bello; Tomas R. Fuentes-Figueroa; Bienvenido M. Vera-Tumbaco; Narciso Fernando Augusto Ayón-Villao

Un canal de distribución es el camino seguido por un producto o servicio para ir desde la fase de producción a la adquisición y consumo, el camino de un canal de distribución lo constituyen una serie de empresas y personas que se denominan intermediarios, que son quienes realizan las funciones de distribución.

El tamaño de los canales se mide por el numero de intermediarios, así el canal (agricultormayorista-minorista-consumidor), (productor-minorista-consumidor) o (agricultor -consumidor); es decir existen canales "largos" y canales "cortos", los canales de distribución cortos conducen a precios de venta al consumidor reducidos. Por el contrario los canales de distribución largos son sinónimos de precios elevados, IFAIN (1999).

La venta del producto final se la realiza en los mercados locales cercanos al sitio de producción con el fin de evitar gastos excesivos por concepto de transportación. La entrega del producto debe realizarse en las primeras horas del día de parte del productor al comprador, puesto que los mayoristas se encargan de distribuir los sacos con pimiento a minoristas y de ahí al consumidor final, completando la cadena de comercialización no solo en el caso de pimiento si no de todo producto tipo perecible (hortalizas y frutas). www.dspace.espol.edu.ec

\section{Resultados.}

La comprobación de las hipótesis se la efectuó a través del desarrollo de la investigación.

La hipótesis general se comprobó y verificó con el análisis de los resultados del desarrollo de la actividad en el cultivo del pimiento, en base a las encuestas que se efectuaron a los cultivadores de esta hortaliza en la Parroquia Puerto Cayo, del Cantón Jipijapa. 


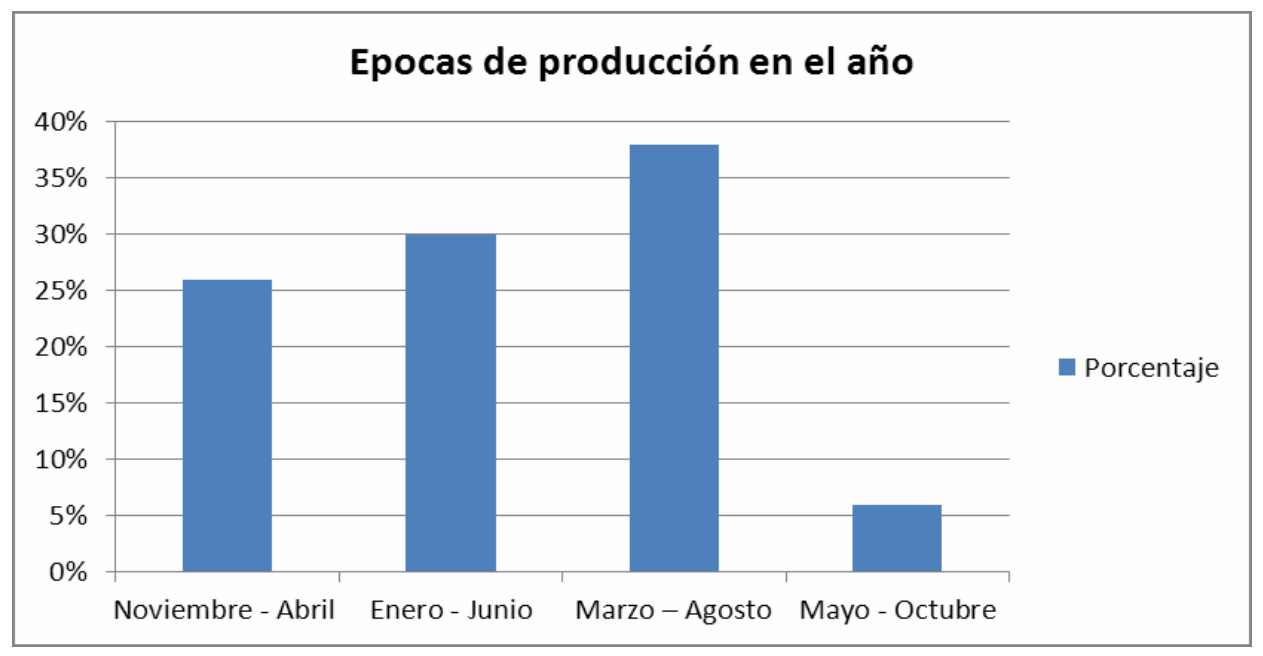

Figura 1. La época de mayor actividad para el cultivo de pimento en la zona es en el primer semestre del año

Es importante indicar que de acuerdo a la encuesta el 26\% de los productores manifestaron que la actividad la realizan en el ciclo que comienza en noviembre, iniciando su producción en enero hasta abril; mientras el $30 \%$ de los agricultores respondieron que inician el ciclo en enero obteniendo la producción a partir de marzo hasta junio; el 38\% indicaron que el ciclo de cultivo lo inician en marzo comenzando la cosecha de pimiento en mayo hasta fines de agosto; finalmente el $6 \%$ de agricultores expresaron que ellos inician el cultivo de pimiento en mayo comenzando la cosecha en julio hasta fines de octubre. Como se muestra en la figura 1.

A través de las encuestas aplicadas a los productores, se comprobó la baja producción en una época del año, así como el mal manejo en la recolección del fruto, incidiendo significativamente en el volumen de producción de pimiento en la zona. 


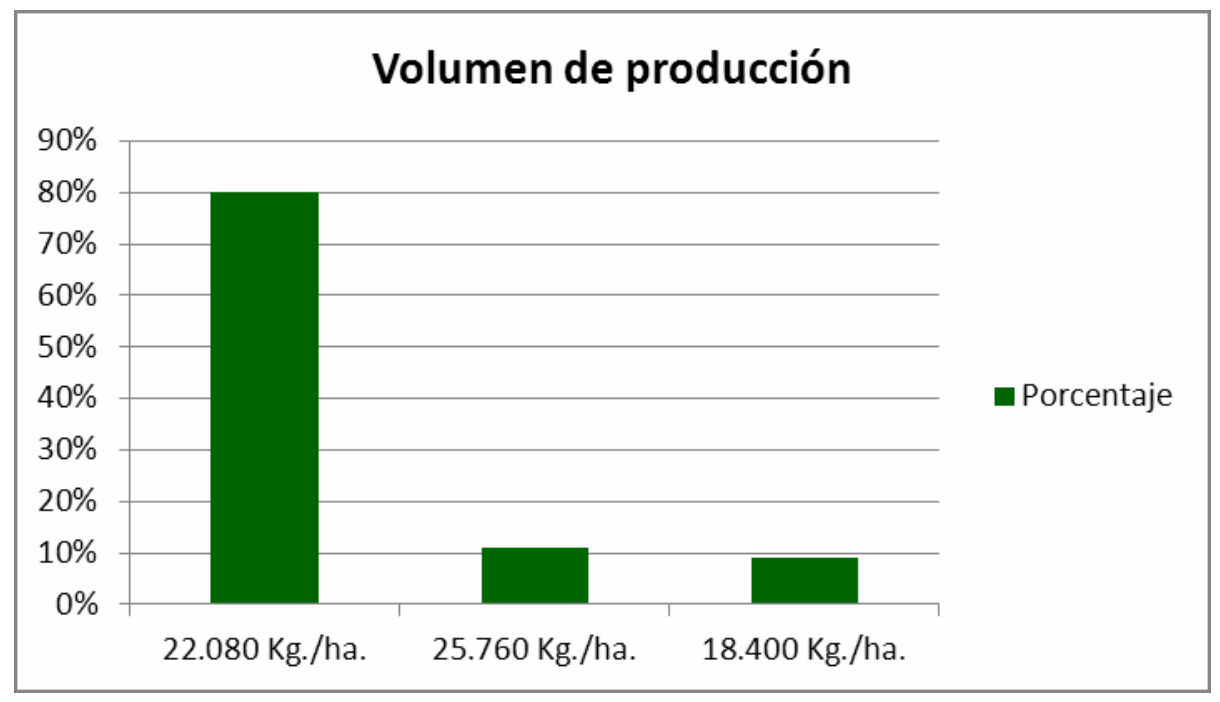

Figura 2. Volumen de producción de pimiento por hectáre

La figura 2, muestra que de la consulta realizada a los agricultores se pudo conocer el volumen de producción de pimiento por hectárea, de ellos resulto: que el $80 \%$ de los horticultores indicaron que la producción de pimiento en su parcela es de $22.080 \mathrm{Kg}$./ha.; el $11 \%$ agricultores manifestaron que es de $25.760 \mathrm{Kg}$./ha.; y el 9\%, dijeron que su producción de pimiento se encuentra en $18.400 \mathrm{Kg} . / \mathrm{ha}$.

Sin embargo a pesar del bajo rendimiento en comparación con otro sector el horticultor si obtiene rentabilidad, esto esta en relación con lo que se invierte, pero no debe ser así, requiere el apoyo institucional para mejorar su volumen de producción.

Mediante las encuestas direccionadas a los productores de esta hortaliza, se comprobó cuando los productores indican que no comercializan a través de centros de acopio, por el contrario para la distribución del producto utilizan tres canales de comercialización que son los que facilitan la venta, distribuyendo el pimiento en todo el país. 


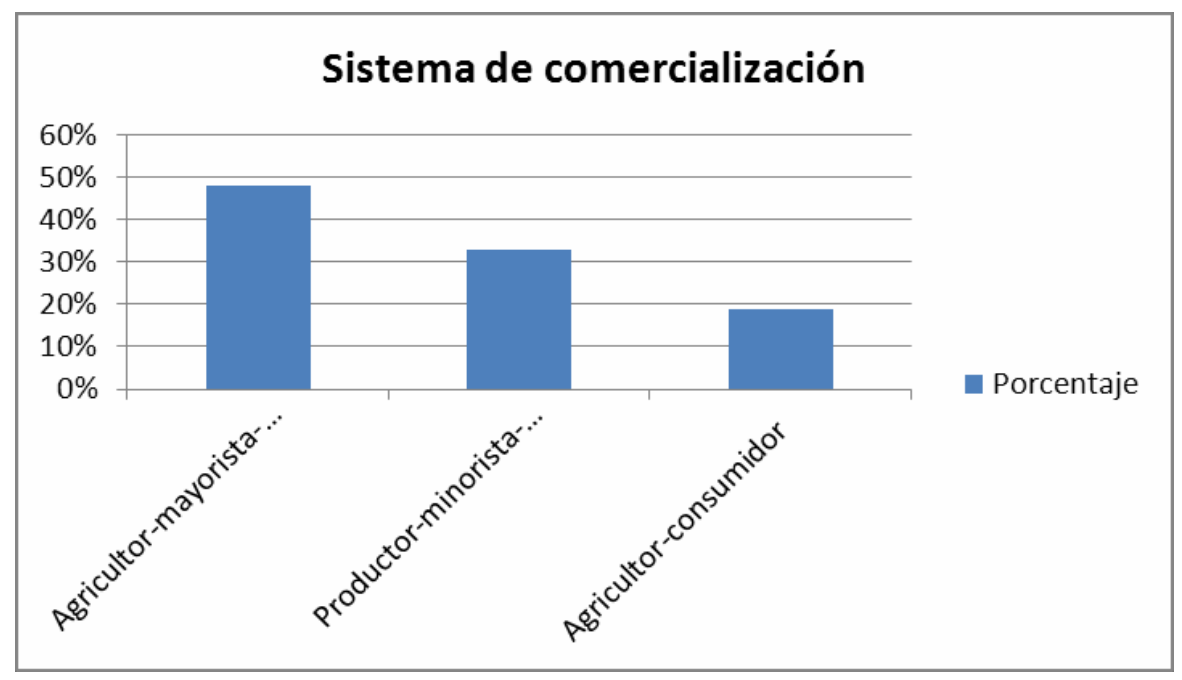

Figura 3. Canal de distribución utilizado para la comercialización del pimiento

Con la finalidad de saber el canal de distribución utilizado para la comercialización del pimiento, se obtuvo la siguiente información de parte de los horticultores, el 48\%, manifestaron que aplican el sistema Agricultor-mayorista-minorista-consumidor, mientras tanto el 33\% de los productores respondieron que el canal que ellos utilizan es el Productor-minorista-consumidor; finalmente el 19\% de los agricultores respondieron que el canal utilizado es Agricultor-consumidor.

Con las encuestas dirigidas a los cultivadores, se la comprobó al deducir que lógicamente el desarrollo de este sector productivo está sujeto a la presencia de varios factores como la experiencia y capacitación en la producción de pimiento, la disponibilidad de los recursos así como el área de siembra; el material vegetal, la existencia de mano de obra, en definitiva el costo de producción así como el precio de venta del pimiento y además otros factores como los naturales que pueden incidir de manera ventajosa o en desventaja a esta actividad productiva. 


\section{Discusión}

Analizando los resultados podemos explicar que la explotación del cultivo de pimiento en la zona de investigación la realizan casi todo el año, pero vale resaltar la época en que la mayoría de los agricultores producen pimiento, la misma que esta comprendida entre los meses de noviembre y agosto. Con esto queda demostrado la época de mayor actividad para el cultivo de pimento en la zona es en el primer semestre del año. En coincidencia con lo establecido en la tabla elaborada por (Holguin Mora, P. y Romero, E. 2002).

Sin embargo hay un 6\% de agricultores que siembra en mayo, esta baja en los cultivadores se debe por la baja en la temperatura por lo que el horticultor no arriesga, debido a que la baja de temperatura tiene su incidencia en el desarrollo vegetativo del cultivo, como se puede observar la producción es irregular en el área. A diferencia de lo manifestado por (Holguin Mora, P. y Romero, E. 2002). que la producción del pimiento para el mercado interno se da la mayor parte en verano. En la Costa es entre Julio y Enero.

Los resultados determinaron que la producción de pimiento oscila en la mayoría de las explotaciones entre los $22.080 \mathrm{Kg} / \mathrm{ha}$. Frente a lo referente que indica que los rendimientos puede alcanzar $48.000 \mathrm{~kg} / \mathrm{ha}$. Respecto a los reportados por www.eluniverso.com (2002 - 2003), y por www.dspace.espol.edu.ec .

Los resultados en lo que se refiere al volumen de producción de pimiento en los recintos tanto en Puerto La Boca como en Cantagallo se mostro irregular, además es baja por unidad de superficie en relación a otras zonas productoras. Con respecto a lo reportados por $\underline{\text { www.dspace.espol.edu.ec }}$ 
Los resultados demuestran que de los horticultores, el $48 \%$ aplican el sistema Agricultormayorista-minorista-consumidor, el 33\% de ellos utilizan el canal Productor-minorista-consumidor; finalmente el 19\% de los agricultores emplean el canal Agricultor-consumidor. De acuerdo con lo logrado por la www.dspace.espol.edu.ec

Cabe destacar que el $19 \%$ que vende su producto directamente al consumidor, siempre van a obtener mejores ingresos económicos a cambio del $48 \%$ aplican el sistema Agricultor-mayoristaminorista-consumidor. De acuerdo con lo que indica IFAIN (1999).

\section{Conclusiones.}

Con la presente investigación se logro demostrar que la época de mayor actividad para el cultivo de pimento en la zona se desarrolla en el primer semestre del año.

Luego de realizar el análisis de los resultados se logro identificar la falta de recursos económicos, del mismo modo el desconocimiento de nuevas tecnologías en el manejo del cultivo, fundamentos que no les permite ampliar el cultivo a mayor superficie, siendo igualmente un impedimento para su desarrollo en los aspectos económico social y cultural para una vida mejor.

El volumen de producción de pimiento en los recintos tanto en Puerto La Boca como en Cantagallo se mostro irregular. Debe tener en cuenta al momento de realizar la cosecha la forma de separar el fruto de la planta, puesto que el mal manejo a más de provocar daños al vegetal causa una baja en el volumen de producción.

En los recintos Puerto La Boca y Cantagallo es superior el número de productores que no reciben capacitación. Asimismo no mantienen una coherencia de las actividades administrativas en 
correspondencia con las actividades prácticas de campo, el agricultor no sabe cuánto invierte realmente y cuanto le retorna como rentabilidad de esta actividad productiva.

El desconocimiento de técnicas de manejo en el cultivo del pimiento no les permite lograr incrementos en la producción y de la misma manera el costo de producción en la zona de investigación es más bajo. Por otro lado el precio del fruto no es fijo debido a que está sujeto a variación, así como también tiende a incrementarse o bajar dependiendo de la oferta y la demanda del producto.

Los horticultores no comercializan sus productos a través de centros de acopio ubicados en el sitio de investigación por cuanto estos no existen. Por lo tanto la gran mayoría de los agricultores utiliza el canal Agricultor-mayorista-minorista-consumidor, indicando como resultado que el producto llega al consumidor con precio elevado. Por el contrario el productor recibe precio bajo en correspondencia al que paga el consumidor.

\section{Bibliografía.}

Holguin Mora, P. y Romero, E. (2002). Estudio de Prefactibilidad para la Producción de Pimiento en la Península de Santa Elena.

IFAIN, (1999), Mercadeo para Proyectos Agroindustriales, Noveno Curso Internacional, Instituto Latinoamericano de Fomento Agroindustrial, Ecuador.

http://www.dspace.espol.edu.ec/bitstream/123456789/474/1/919.pdf En línea Ecuador consultado el 11 de abril de 2014.

Linares O., Heladio, (2004), Cultivo del Pimiento Morron. Curso teórico práctico.

Tuárez Choéz, L. (2009). Producción y comercialización del pimiento (Capsicum annum), e incidencia socioeconómica de Puerto Cayo, Jipijapa. Universidad Estatal del Sur de Manabí.

www.eluniverso.com $(2002-2003)$. 